\title{
Generation of superparamagnetism in metallic $\alpha$-iron by swift heavy ion irradiation
}

E. Kuzmann ${ }^{1 *}$, S. Stichleutner ${ }^{1,2}$, Z. Homonnay ${ }^{1}$, K. Havancsák ${ }^{3}$, C. U. Chisholm ${ }^{4}$, M. ElSharif $^{4}$, V. A. Skuratov ${ }^{5}$, A. Nakanishi ${ }^{6}$ and K. Nomura ${ }^{7}$

${ }^{1}$ Institute of Chemistry, Eötvös Loránd University, 1117, Budapest, Pázmány Péter sétany 1/A, Hungary

${ }^{2}$ Centre for Energy Research, Hungarian Academy of Sciences, 1121 Budapest, Konkoly Thege Miklós út 29-33, Hungary

${ }^{3}$ Institute of Physics, Eötvös Loránd University, 1117, Budapest, Pázmány Péter sétany 1/A, Hungary

${ }^{4}$ Surface Technology Research Group, School of Engineering and the Built Environment, Glasgow Caledonian University, Cowcaddens Road G0 4 BA, Scotland, UK

${ }^{5}$ Flerov Laboratory of Nuclear Reactions, Joint Institute for Nuclear Research, JINR, Joliot-Curie 6, 141980, Dubna, Moscow region, Russia

${ }^{6}$ Department of Physics, Shiga University of Medical Science, Seta, 520-21, Otsu, Shiga, Japan

${ }^{7}$ Department of Applied Chemistry, Science and Engineering, Meiji University, 1 Chome1 Kanda Surugadai, Chiyoda, Tokyo 101-8301, Japan

*Corresponding author, e-mail:kuzmann@caesar.elte.hu phone:36204467873 Keywords: Superparamagnetic iron, Amorphous iron, ${ }^{57} \mathrm{Fe}$ conversion electron Mössbauer spectroscopy, Swift heavy ion irradiation, Electrodeposition 


\begin{abstract}
${ }^{57} \mathrm{Fe}$ conversion electron Mössbauer spectroscopy was used to study the effect of swift heavy ion irradiation on electrochemically deposited metallic pure $\alpha$-iron. We succeeded in preparing superparamagnetic iron by irradiating the electrochemically prepared thin $\alpha$-iron films using $247 \mathrm{MeV} \mathrm{Kr}$ ions with a fluence of $1 \times 10^{13}$ ion $\mathrm{cm}^{-2}$ which converted $50 \%$ of crystalline $\alpha$-iron into amorphous and superparamagnetic phases, the latter being $>20 \%$. The results are discussed in terms of the thermal spike model for the formation of the amorphous phase which could be essential for the formation of superparamagnetic iron.
\end{abstract}

\title{
1. Introduction
}

In our previous works we performed Mössbauer spectroscopic and XRD studies on the radiation effect of $246 \mathrm{MeV} \mathrm{Kr}, 470 \mathrm{MeV} \mathrm{Xe}$ and $710 \mathrm{MeV} \mathrm{Bi}$ ions on electrochemically deposited iron thin films [1,2]. It was found that as a result of irradiation partial amorphisation of iron took place in the electrochemically deposited crystalline ferromagnetic $\alpha$-iron coatings. The relative amount of the ferromagnetic amorphous phase increased with both ion energy and ion mass as well as with the fluence of irradiation. Besides ferromagnetic amorphous iron, another iron phase was also identified in the room temperature Mössbauer spectra of the swift heavy ion irradiated samples and was associated with superparamagnetic iron. 
Superparamagnetism [3-5] is a relaxation phenomenon observed below the Curie temperature in single domain ferromagnetic or ferrimagnetic nanoparticles/nanograins [613] $(1-10 \mathrm{~nm})$ where magnetisation can randomly flip between the opposite directions of the easy axis, provided that the thermal energy is sufficient to overcome the corresponding energy barrier. The mean time between flips is known as the Néel relaxation time [14-19]. Smaller nanoparticles/nanograins can be expected to exhibit a larger frequency of superparamagnetic relaxation. When the time window of the experimental method used to determine the magnetisation of superparamagnetic nanoparticle/nanograin ensembles is significantly longer than the Néel relaxation time, the detected magnetisation tends to average to zero, which is a typical characteristic of the superparamagnetic state [20]. Superparamagnetic iron was originally found to form under natural circumstances in samples from the moon surface [21-23]. Superparamagnetic inclusions of metallic iron, some tens of $\mathrm{nm}$ in size, were formed in the amorphous rims of lunar regolith grains from solar-wind-reduced indigenous iron silicates by either radiation-induced lattice rearrangement, or micrometeorite-induced vaporisation and recondensation, or both [24].

However, a process to obtain superparamagnetism in metallic pure $\alpha$-iron is still unknown. In this work we extended our study on the radiation effect of swift heavy ions on electrochemically deposited iron thin films by conversion electron Mössbauer measurements carried out at low temperature, in order to confirm the presence of superparamagnetic iron in our irradiated samples.

\section{Experimental}


The $\alpha$-iron coatings were prepared using a constant current technique with a continuous flow electrodeposition cell plating system. The thin films were deposited on electro-polished pure copper substrates using the following electrolyte composition: $\mathrm{FeSO}_{4} 0.2 \mathrm{~mol} \mathrm{dm}^{-3}$, Na-gluconate $120 \mathrm{~g} \mathrm{dm}^{-3}$ and peptone $0.1 \mathrm{~g} \mathrm{dm}^{-3}$. The electrolyte was operated at $60{ }^{\circ} \mathrm{C}$ with a $\mathrm{pH}=7.0$ and plating times of $20 \mathrm{~min}$. were used.

The swift heavy ion irradiation of electroplated $\alpha$-iron was carried out with 246 MeV energy ${ }^{86} \mathrm{Kr}^{8+}$ ions with a fluence of $1 \times 10^{13}$ ion $\mathrm{cm}^{-2}$ at room temperature, at a current density of $0.5 \mu \mathrm{A} \mathrm{cm}^{-2}$ and at a pressure of about $10^{-3} \mathrm{~Pa}$, at the U-400 cyclotron of the Flerov Laboratory of Nuclear Reactions, JINR, Dubna, Russia.

Conversion electron Mössbauer measurements of as-deposited and irradiated $\alpha$ iron samples were performed by conventional Mössbauer spectrometers (WISSEL) with flowing gas $\left(96 \% \mathrm{He}, 4 \% \mathrm{CH}_{4}\right.$ at $293 \mathrm{~K}$ and purified helium at $15 \mathrm{~K}$ ) proportional counters and ${ }^{57} \mathrm{Co}(\mathrm{Rh})$ sources of $1.85 \mathrm{GBq}$ activity, at room temperature and at $15 \mathrm{~K}$. For the $15 \mathrm{~K}$ CEMS measurement a cryostat (IwataniCryoMini D310) and counter assembly was used $[25,26]$. The information can be obtained mainly from sample depths less than $100 \mathrm{~nm}[27,28]$. Isomer shifts are given relative to $\alpha$-iron. The evaluation of Mössbauer spectra was performed by least-square fitting of the lines using the MOSSWINN code [29]. During the fitting procedure the amplitudes of $2^{\text {nd }}$ and $5^{\text {th }}$ lines of sextets were set to be independent, while independent linewidths for $1^{\text {st }}$ and $6^{\text {th }}, 2^{\text {nd }}$ and $5^{\text {th }}, 3^{\text {rd }}$ and $4^{\text {th }}$ line pairs were constrained.

\section{Results and discussion}


${ }^{57} \mathrm{Fe}$ conversion electron Mössbauer spectra of electrodeposited $\alpha$-iron samples: (a) spectrum for as-deposited sample recorded at $293 \mathrm{~K}$, (b) spectrum for sample irradiated with $246 \mathrm{MeV}$ energy $\mathrm{Kr}$ ions with a fluence of $1 \times 10^{13}$ ion $\mathrm{cm}^{-2}$ recorded at $293 \mathrm{~K}$, (c) spectrum for irradiated sample recorded at $15 \mathrm{~K}$ are shown in Fig. 1. The Mössbauer parameters are shown in Table I. The presented numerical results derived from Fig. 1a,b slightly deviate from those published in [1] since we applied a different model for the evaluation of the spectra, resulting in a better quality of the fits in the present cases.

We investigated metallic pure $\alpha$-iron in the form of thin electrodeposited films. The room temperature spectrum of the non-irradiated, as-deposited sample shown in Fig. 1(a) consists of a dominating sextet (S1) with narrow spectral lines and a small intensity doublet (D1). Sextet S1 is the fingerprint of the crystalline $\alpha$-iron which has a bcc structure [30]. This was also confirmed by XRD measurements which revealed solely the presence of the crystalline $\alpha$-iron phase in the electrodeposited sample. This result is consistent with the type of structure to be expected from the electrodeposition of iron. Doublet D1 can be attributed to an iron oxy-hydroxide phase based on its characteristic Mössbauer parameters [30]. As X-ray diffractograms did not exhibit the existence of this phase it can be interpreted to be a thin layer (circa $10 \mathrm{~nm}$ ) which has formed on the surface of the electrodeposited $\alpha$-iron. This phase can be explained by the corrosion of the surface of the electrodeposited $\alpha$-iron by air after electrodeposition.

Fig. 1(b) shows the room temperature spectrum of the irradiated sample, which is decomposed into two sextets (S1 and S2), a doublet (D1) and a singlet (P1). The 
corresponding Mössbauer parameters are shown in Table I. This decomposition was found to be the optimal for the spectrum fitting at minimal number of components, which is consistent with those obtained previously on swift heavy ion irradiated $\alpha$-iron thin films [1,2]. We used a broad singlet instead of a doublet in [1] for component P1 based on the works of $[31,32]$. Sextet $\mathrm{S} 1$ again relates to the crystalline $\alpha$-iron phase. Sextet S2 has very broad spectral lines which are typical of amorphous phases. This component can be considered as a superposition of a number of magnetically split sub-spectra belonging to iron atoms being in slightly different microenvironments. The Mössbauer parameters of sextet S2 are an excellent match for those of amorphous iron phase $[33,34]$ and thus sextet S2 was assigned to amorphous iron phase [1,2]. Doublet D1 again corresponds to the iron-oxy-hydroxide phase. Singlet P1 exhibits a very broad line and the value of the isomer shift of singlet $\mathrm{P} 1$ shown in Table $\mathrm{I}$ is characteristic of metallic bcc iron. Taking into consideration the phase composition of the electrodeposited sample, this excludes the assignment of singlet P1 to any other iron-bearing phase other than one of pure iron. However, bcc iron is in a ferromagnetic state, resulting in a typical magnetically split pattern such as shown by sextet S1. Surprisingly we observed no magnetic splitting in this case. This indicates that the iron atoms represented by singlet P1 are in a state which can only be explained by the presence of a superparamagnetic iron phase. The broad spectral line of singlet $\mathrm{P} 1$ is a typical relaxation spectrum of a superparamagnetic phase. The contour of singlet P1 illustrated in Fig. 1(b) corresponds closely with that predicted for a superparamagnetic iron phase [31,32]. Irradiation of the electrodeposited $\alpha$-iron samples with $246 \mathrm{MeV}$ energy $\mathrm{Kr}$ ions with a fluence of $1 \times 10^{13}$ ion $\mathrm{cm}^{-2}$ has resulted in the transformation of about $50 \%$ of the crystalline $\alpha$-iron phase into amorphous and 
superparamagnetic iron phases, the occurrence of the latter being found to be higher than 20\%. The corresponding XRD patterns of the irradiated samples, which are very similar to those we reported earlier [1,2], show the presence of crystalline iron only in $\alpha-\mathrm{Fe}$ (bcc) form, which is consistent with occurrence of superparamagnetic iron.

As can be seen from Fig. 1(c), the CEM spectrum recorded at $15 \mathrm{~K}$ is decomposed into two sextets (S1 and S2) and a doublet (D1). The envelope of this spectrum is similar to that of the spectrum of the non-irradiated, as-deposited sample recorded at room temperature (Fig. 1(a)). No singlet component could be fitted in the spectrum and all three spectral components (S1, S2, D1) were assigned correspondingly to the room temperature spectrum of the irradiated sample (Fig. 1(b)). The isomer shift of the different sub-spectra exhibits regular temperature dependence [35], while quadrupole splitting and hyperfine field at different temperatures may also be consistent with their regular temperature behavior [36] as they appear to be overlapping within a wide confidence interval considering the standard deviation values shown in Table I. However we observed significant changes in the spectral areas of the different components in comparison with those detected in the room temperature CEM spectrum. Singlet P1, which we expected to appear at $0.13 \mathrm{~mm} \mathrm{~s}^{-1}$ isomer shift value according to the second order Doppler shift, did not appear in the Mössbauer spectrum and the relative area of sextet $\mathrm{S} 1$ belonging to crystalline $\alpha$-iron increased. Taking into account the temperature dependence of the Lamb-Mössbauer factors of the phases, the increment of spectral area of sextet $\mathrm{S} 1$ at $15 \mathrm{~K}$ corresponds to the spectral area of singlet $\mathrm{P} 1$ at room temperature. Consequently, the changes between the $15 \mathrm{~K}$ and room temperature CEM spectra of the irradiated sample reflect the transformation of the room temperature superparamagnetic 
iron phase into ferromagnetic $\alpha$-iron phase at $15 \mathrm{~K}$. This confirms the correct assignment of superparamagnetic iron phase at room temperature. Note that the above mentioned transformation is inconsistent with the assignment of singlet P1 to a paramagnetic amorphous state. Furthermore, its isomer shift value is lower than expected for amorphous iron [33,34]. The changes between the Mössbauer spectra recorded at $293 \mathrm{~K}$ and $15 \mathrm{~K}$ correspond well to a superparamagnetic relaxation, thus confirming the presence of superparamagnetic iron in our irradiated electrodeposited $\alpha$-iron samples.

Our results suggest that the presence of amorphous iron could be essential to the creation of the superparamagnetic phase. Amorphous iron occurs when the irradiation induces the formation of thermal spikes [37,38], which result in melting and rapid quenching of matter along the path of the ion. The matter temperature along the ion path may significantly exceed the melting point and a liquid cylinder of a few nanometres in diameter described as a thermal spike is formed, which cools down within $10^{-11} \mathrm{~s}$ leading to a cooling rate of $10^{14} \mathrm{~K} \mathrm{~s}^{-1}$. The development of an amorphous matrix structure is then the result of the superposition of individual amorphous regions [37-39]. From our observations we presume that the superparamagnetic metallic iron grains are formed at the boundary of the thermal spike regions and we are of the opinion that the amorphous iron matrix surrounding the superparamagnetic iron grains probably has a significant influence on the grain size and the stability of the superparamagnetic phase. The grain size of the superparamagnetic iron is limited to the small distance between the neighbouring amorphous regions and these, regions probably overlap with increasing irradiation fluence finally leading to a reduction of the superparamagnetic iron phase in direct accordance with our experimental observations [1,2]. Assuming that the 
superparamagnetic component is associated with bcc iron whose first order cubic anisotropy energy density is $K_{l}=4.5 \times 10^{4} \mathrm{~J} \mathrm{~m}^{-3}$ [40] division of room temperature $k_{B} T$ with $K_{l}$ gives an approximate upper limit of circa 5-6 $\mathrm{nm}$ for the size of the superparamagnetic iron grains.

The existing magnetic state below the superparamagnetic transition may also be influenced by the effect of a collective state (like superferromagnetism [41-44]) due to the ferromagnetic metallic iron matrix where non-negligible inter-particle interactions might be present. However, this doesn't affect our finding that superparamagnetic iron phase exists at room temperature.

\section{Conclusion}

We have successfully shown that superparamagnetism can be developed in $\alpha$-iron in the presence of an amorphous matrix generated by irradiation. Our results suggest that the presence of an amorphous phase could be essential for the formation of superparamagnetic iron. We can conclude from our results that the formation of the superparamagnetic iron phase takes place in a similar manner to that found in earlier mentioned lunar samples [21-24]. In both cases the phenomenon occurs due to irradiation with the simultaneous formation of an amorphous phase but in the case of the lunar samples superparamagnetism is the result of chemical reduction due to the solar wind irradiation.

\section{Acknowledgements}


This work was supported by the Hungarian National Science Foundation (OTKA, projects K68135 and K111692) and by the Hungarian Academy of Sciences (project EAI-2009/003-10030).

\section{References}

[1] E. Kuzmann, S. Stichleutner, K. Havancsák, M. R. El-Sharif, C. U. Chisholm, O. Doyle, V. Skuratov, K. Kellner, Gy. Dóra, Z. Homonnay, and A. Vértes, Radiat. Phys. Chem. 75, 741 (2006).

[2] S. Stichleutner, E. Kuzmann, K. Havancsák, A. Huhn, M. R. El-Sharif, C. U. Chisholm, O. Doyle, V. Skuratov, Z. Homonnay, and A. Vértes, Radiat. Phys. Chem. 80, 471 (2011).

[3] C. P. Bean and J. D. Livingston, J. Appl. Phys. 30, 120S (1959).

[4] A. P. Guimarães, Principles of Nanomagnetism (Springer, Berlin, 2009).

[5] J. M. D. Coey, Magnetism and Magnetic Materials (Cambridge University Press, Cambridge, 2010).

[6] F. Bødker, S. Mørup, C. A. Oxborrow, S. Linderoth, M. B. Madsen, and J. W. Niemansverdriet, J. Phys. Condens. Matter 4, 6555 (1992).

[7] Q. A. Pankhurst and R. J. Pollard, J. Phys. Condens. Matter 5, 8487 (1993).

[8] P. Brahma, S. Banerjee, D. Das, P. K. Mukhopadhyay, S. Chatterjee, A. K. Nigam, and D. Chakravorty, J. Magn. Magn. Mater. 246, 162 (2002). 
[9] R. G. Wilks, E. Z. Kurmaev, J. C. Pivin, A. Hunt, M. V. Yablonskikh, D. A. Zatsepin, A. Moewes, S. Shin, P. Palade, and G. Principi, J. Phys. Condens. Matter 17, 7023 (2005).

[10] M. P. Fernández, D. S. Schmool, A. S. Silva, M. Sevilla, A. B. Fuertes, P. Gorria, and J. A. Blanco, J. Non-Crys. Solids 354, 5219 (2008).

[11] T. Jafari, A. Simchi, and N. Khakpash, J. Colloid Interf. Sci. 345, 64 (2010).

[12] I. Sakamoto, S. Purwanto, M. Koike, S. Honda, N. Hayashi, Y. Miyamoto, H. Wakabayshi, T. Toriyama, and K. Nomura, J. Phys. Conf. Ser. 217, 012099 (2010).

[13] K. Nomura and H. Reuther, J. Radioanal. Nucl. Chem. 287, 341 (2011).

[14] L. Néel, Ann. Geophys. 5, 99 (1949).

[15] W. F. Brown, Phys. Rev. 130, 1677 (1963).

[16] M. Knobel, W. C. Nunes, L. M. Socolovsky, E. De Biasi, J. M. Vargas, and J. C. Denardin, J. Nanosci. Nanotechnol. 8, 2836 (2008).

[17] S. Bedanta and W. Kleemann, J. Phys. D: Appl. Phys. 42, 013001 (2009).

[18] S. Morup, M. F. Hansen, and C. Frandsen, Beilstein J. Nanotechnol. 1, $182(2010)$.

[19] A. G. Kolhatkar, A. C. Jamison, D. Litvinov, R. C. Willson, and T. R. Lee, Int. J. Mol. Sci. 14, 15977 (2013).

[20] L. Lopez-Diaz, L. Torres, and E. Moro, Phys. Rev. B 65, 224406 (2002).

[21] S. S. Hafner, in Mössbauer Spectroscopy, edited by U. Gonser (Springer, Berlin, 1975). 
[22] R. M. Housley, R. W. Grant, and N. E. Paton, Geochim. Cosmochim. Acta, Suppl. 4, 2737 (1973).

[23] R. M. Housley, E. H. Cirlin, N. E. Paton, and I. B. Goldberg, Geochim. Cosmochim. Acta, Suppl. 5, 2623 (1974).

[24] J.F. Kerridge, in Abstracts of the 25th Lunar and Planetary Science Conference (Houston, 14-18 March 1994).

[25] K. Fukumura, R. Katano, T. Kobayashi, A. Nakanishi and Y. Isozumi, Nucl. Instrum. Methods A 301, 482 (1991).

[26] K. Fukumura, A. Nakanishi, T. Kobayashi, R. Katano and Y. Isozumi, Nucl. Instrum. Methods B 61, 127 (1991).

[27] T. Kobayashi, K. Fukumura and A. Nakanishi, Nucl. Instrum. Methods B 76, 204 (1993).

[28] Y. Yonekura, T. Toriyama, J. Itoh and K. Hisatake, Hyperfine Interact. 15/16, 1005 (1983).

[29] Z. Klencsár E. Kuzmann and A. Vértes, J. Radioanal. Nucl. Ch. 210, 105 (1996).

[30] J. G. Stevens and V. E. Stevens, Mössbauer Effect Data Index (Adam Hilger, London, 1965-1975).

[31] A. M. Afanas'ev and M. A. Chuev, J. Exp. Theor. Phys. Lett. 74, 107 (2001).

[32] A. M. Afanas'ev and M. A. Chuev, J. Exp. Theor. Phys. Lett. 77, 415 (2003). 

353, 414 (1991).

[34] G. J. Long, D. Hautot, Q. A. Pankhurst, D. Vandormael, F. Grandjean, J. P. Gaspard, V. Briois, T. Hyeon, and K. S. Suslick, Phys. Rev. B 57, 10716 (1998).

[35] A. Vértes, L. Korecz, and K. Burger, Mössbauer Spectroscopy

(Akadémiai Kiadó, Budapest, 1979).

[36] E. Kuzmann, S. Nagy and A. Vértes, Pure Appl. Chem. 75, 801 (2003).

[37] M. Toulemonde and Ch. Dufour, Phys. Rev. B 46, 14362 (1992).

[38] M. Toulemonde, E.Paumier, and Ch. Dufour, Radiat. Eff. Defects Sol. 126, 201 (1993).

[39] Z. G. Wang, Ch. Dufour, S. Euphrasie, and M. Toulemonde, Nucl. Instrum. Methods B 209, 194 (2003).

[40] J. Ye, W. He, Q. Wu, H.-L. Liu, X.-Q. Zhang, Z.-Y. Chen and Z.-H. Cheng, Sci. Rep. 3, 2148 (2013).

[41] S. Morup, M. B. Madsen, J. Franck, J. Villadsen, and C. J. W. Koch, J. Magn. Magn. Mater. 40, 163 (1983).

[42] S. Morup, Hyperfine Interact. 60, 959 (1990).

[43] S. Morup, Hyperfine Interact. 90, 171 (1994).

[44] S. Bedanta, T. Eimüller, W. Kleemann, J. Rhensius, F. Stromberg, E. Amaladass, S. Cardoso, and P. P. Freitas, Phys. Rev. Lett. 98, 176601 (2007). 

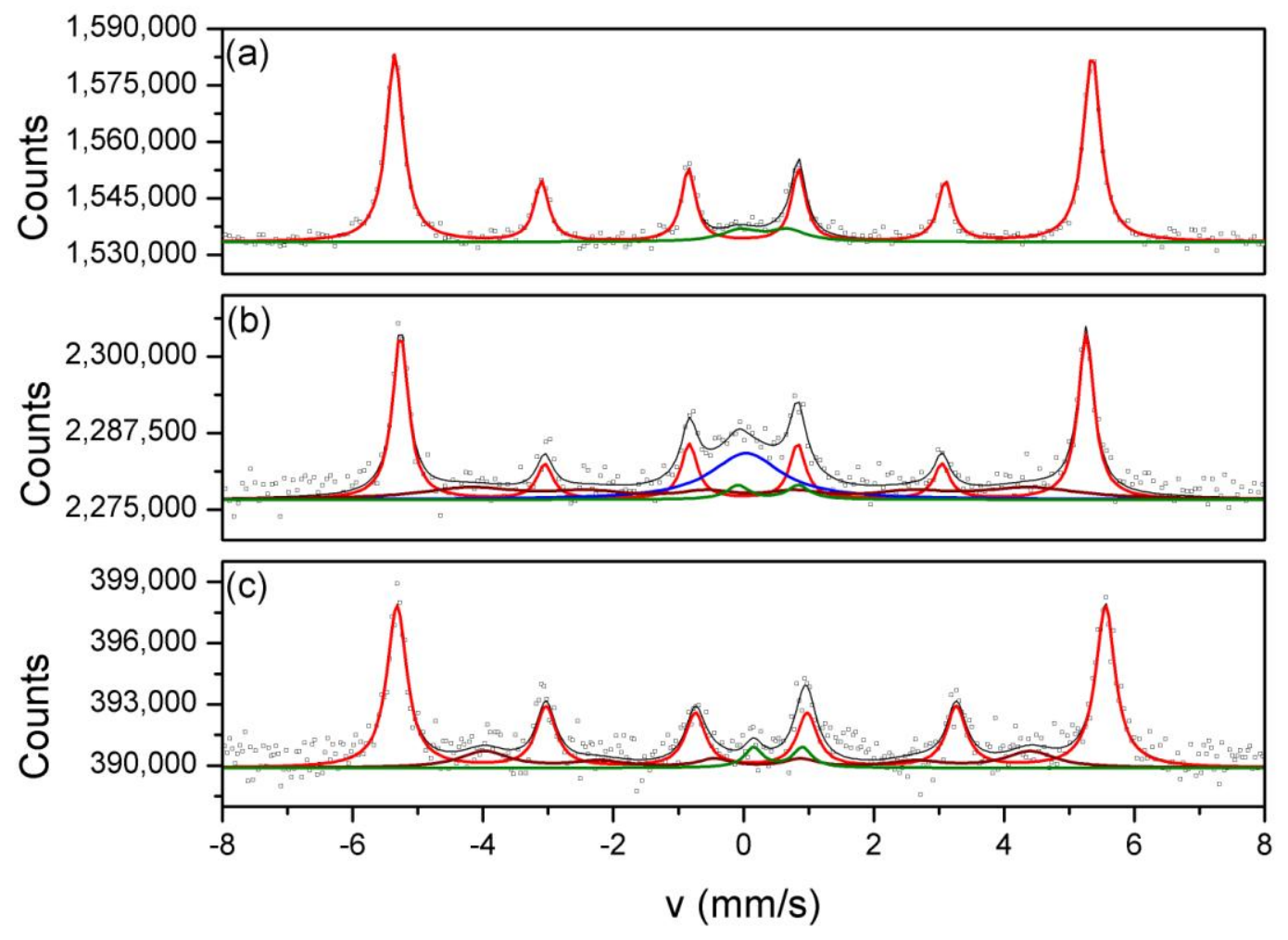

FIG. 1. ${ }^{57} \mathrm{Fe}$ conversion electron Mössbauer spectra of electrodeposited $\alpha$-iron samples: (a) spectrum for as-deposited sample recorded at $293 \mathrm{~K}$, (b) spectrum for sample irradiated with $246 \mathrm{MeV}$ energy $\mathrm{Kr}$ ions with a fluence of $1 \times 10^{13}$ ion $\mathrm{cm}^{-2}$ recorded at $293 \mathrm{~K}$, (c) spectrum for irradiated sample recorded at $15 \mathrm{~K}$. 


\begin{tabular}{|c|c|c|c|}
\hline $\begin{array}{l}\text { Mössbauer } \\
\text { parameters }\end{array}$ & Non-irradiated & $\begin{array}{l}\mathrm{Kr}, 1 \times 10^{13} \text { ion } \mathrm{cm}^{-2}, \\
\text { measured at } 293 \mathrm{~K}\end{array}$ & $\begin{array}{c}\mathrm{Kr}, 1 \times 10^{13} \text { ion } \mathrm{cm}^{-2}, \\
\text { measured at } 15 \mathrm{~K}\end{array}$ \\
\hline $\begin{array}{c}\delta \\
\left(\begin{array}{c}\text { doublet D1) } \\
\mathrm{mm} \mathrm{s}^{-1}\end{array}\right.\end{array}$ & $0.31 \pm 0.019$ & $0.38 \pm 0.025$ & $0.53 \pm 0.021$ \\
\hline $\begin{array}{c}\Delta \\
\text { (doublet D1), } \\
\mathrm{mm} \mathrm{s}^{-1}\end{array}$ & $0.73 \pm 0.039$ & $0.93 \pm 0.043$ & $0.75 \pm 0.041$ \\
\hline $\begin{array}{c}\delta \\
\text { (sextet S1) } \\
\mathrm{mm} \mathrm{s}^{-1}\end{array}$ & $0 \pm 0.010$ & $0 \pm 0.010$ & $0.12 \pm 0.011$ \\
\hline $\begin{array}{c}B \\
\text { (sextet } \mathrm{S} 1) \text {, } \\
\mathrm{T}\end{array}$ & $33.0 \pm 0.25$ & $32.7 \pm 0.32$ & $33.7 \pm 0.35$ \\
\hline $\begin{array}{c}\delta \\
\text { (sextet S2) } \\
\mathrm{mm} \mathrm{s}^{-1}\end{array}$ & - & $0.1 \pm 0.015$ & $0.21 \pm 0.016$ \\
\hline $\begin{array}{c}B \\
\text { (sextet S2), } \\
\mathrm{T} \\
\delta\end{array}$ & - & $26.7 \pm 0.61$ & $26.0 \pm 0.62$ \\
\hline $\begin{array}{l}\text { (singlet P1) } \\
\mathrm{mm} \mathrm{s}^{-1}\end{array}$ & - & $0.04 \pm 0.011$ & 一 \\
\hline $\begin{array}{c}A \\
\text { (doublet D1), } \\
\%\end{array}$ & $7.7 \pm 2.8$ & $3.8 \pm 2.5$ & $5.1 \pm 2.6$ \\
\hline $\begin{array}{c}A \\
(\text { sextet } \mathrm{S} 1) \\
\%\end{array}$ & $92.3 \pm 2.0$ & $51.0 \pm 2.2$ & $75.6 \pm 2.1$ \\
\hline $\begin{array}{c}A \\
\text { (sextet S2), } \\
\%\end{array}$ & - & $24.6 \pm 3.1$ & $19.4 \pm 2.8$ \\
\hline $\begin{array}{c}A \\
\text { (singlet P1), } \\
\% \\
\end{array}$ & - & $20.6 \pm 2.2$ & 一 \\
\hline
\end{tabular}

TABLE I. Mössbauer parameters relating to the ${ }^{57} \mathrm{Fe}$ conversion electron Mössbauer spectra for electrodeposited $\alpha$-iron samples. $\delta, \Delta, B$ and $A$ denote isomer shift, quadrupole splitting, effective magnetic induction and spectral area, respectively. Linewidths, $W$, of the different sub-spectra were obtained to vary between the following values for $W_{S 1}=0.29-0.36 \pm 0.05 \mathrm{~mm} \mathrm{~s}^{-1}, \quad W_{D I}=0.39-0.69 \pm 0.11 \mathrm{~mm} \mathrm{~s}^{-1}, W_{S 2}=0.9-1.8 \pm 0.21 \mathrm{~mm} \mathrm{~s}^{-1}$ (for the different lines of sextet $\mathrm{S} 2$ ) and $W_{P I}=1.31 \pm 0.08 \mathrm{~mm} \mathrm{~s}^{-1}$, respectively. 